\title{
Fibrosis-4 index at diagnosis is associated with all-cause mortality in patients with microscopic polyangiitis and granulomatosis with polyangiitis
}

Hee Jin Park', Jun Yong Park 2,3 , Seung Min Jung ${ }^{4}$, Jason Jungsik Song ${ }^{4,5}$, Yong-Beom Park ${ }^{4,5}$ and Sang-Won Lee L, $^{*}$ (D)

\begin{abstract}
Background: The fibrosis-4 index (FIB-4) has been reported to be associated with all-cause mortality in several chronic diseases. In this study, we investigated whether at diagnosis could be associated with all-cause mortality in patients with microscopic polyangiitis (MPA) and granulomatosis with polyangiitis (GPA).

Methods: We retrospectively reviewed the medical records of 132 MPA and GPA patients without chronic liver diseases. Conventional risk factors included old age ( $\geq 65$ years), male gender, diabetes mellitus (DM) and hypertension (HTN) at diagnosis, and disease-related risk factor included GPA, antineutrophil cytoplasmic antibody, Birmingham vasculitis activity score (BVAS) and five factor score (FFS (2009)). The cut-off of FIB-4 for significant liver fibrosis (S2-4) was set at 1.45 .

Results: The mean age was 57.2 years and 27 patients (20.5\%) had significant liver fibrosis (FIB-4 $\geq 1.45$ ). Fifteen patients (11.4\%) died during follow-up. In the univariable Cox Hazards model, age $\geq 65$ years (Hazard ratio (HR) 5.055), DM (HR 3.446), HTN (HR 4.611), FFS (2009) $\geq 2$ (HR 4.849) and FIB-4 $\geq 1.45$ (HR 9.958) at diagnosis were significantly associated with all-cause mortality. In the multivariable Cox Hazards model, only FIB-4 at diagnosis $\geq 1.45$ (HR 6.253, 95\% confidence interval 1.398, 27.963) was associated with all-cause mortality during the follow-up in patients with MPA and GPA.
\end{abstract}

Conclusions: FIB-4 at diagnosis $\geq 1.45$ is an independent predictor of all-cause mortality during follow-up in patients with MPA and GPA, and furthermore its predictive potential is higher than those of conventional and AAV-related risk factors for all-cause mortality.

Keywords: Microscopic polyangiitis, Granulomatosis with polyangiitis, FIB-4, Mortality

\section{Background}

Antineutrophil cytoplasmic antibody (ANCA)-associated vasculitis (AAV) is a group systemic vasculitides, which can affect small vessels ranging from capillaries to intraparenchymal arterioles and venules of almost all the

\footnotetext{
* Correspondence: sangwonlee@yuhs.ac

${ }^{4}$ Division of Rheumatology, Department of Internal Medicine, Yonsei University College of Medicine, 50-1 Yonsei-ro, Seodaemun-gu, Seoul 03722, Republic of Korea

${ }^{5}$ Institute for Immunology and Immunological Diseases, Yonsei University College of Medicine, 50-1 Yonsei-ro, Seodaemun-gu, Seoul 03722, Republic of Korea

Full list of author information is available at the end of the article
}

organs [1]. AAV consists of three variants, such as microscopic polyangiitis (MPA), granulomatosis with polyangiitis (GPA) and eosinophilic granulomatosis with polyangiitis (EGPA) [1, 2]. MPA and GPA mainly present similar pulmonary and renal symptoms despite different genetic and antigenic backgrounds, whereas EGPA often exhibit both allergic and necrotising vasculitic features [1-3].

Since AAV can bring out an amount of inflammatory burden and damage in major organs including lungs, kidneys and heart, AAV can be occasionally fatal. So far, there have been diverse reports regarding all-cause

(c) The Author(s). 2019 Open Access This article is distributed under the terms of the Creative Commons Attribution 4.0 International License (http://creativecommons.org/licenses/by/4.0/), which permits unrestricted use, distribution, and reproduction in any medium, provided you give appropriate credit to the original author(s) and the source, provide a link to the Creative Commons license, and indicate if changes were made. The Creative Commons Public Domain Dedication waiver (http://creativecommons.org/publicdomain/zero/1.0/) applies to the data made available in this article, unless otherwise stated. 
mortality in patients with AAV from different ethnic and geographic backgrounds: one year-cumulative patient survival rates were from 82 to $95 \%$ in Western countries [4], and that was reported to be $79.1 \%$ in Japan [5]. In Korean patients with AAV, 10-year cumulative patient survival rate was estimated up to $92.8 \%$ [6].

Renal and pulmonary involvements and five factor score (FFS (2009)) $\geq 2$ at diagnosis of AAV have been reported as AAV-related risk factors for all-cause mortality $[6,7]$, together with conventional risk factors for allcause mortality among the general population including age, male gender, diabetes mellitus (DM) and hypertension (HTN) [8]. Although infectious or immunosuppressive drug-related causes are major aetiologies of death in AAV patients, identifying predictors of all-cause mortality at diagnosis in immunosuppressive drug-naïve patients may have clinical implications in the real settings.

The fibrosis-4 index (FIB-4), which is calculated based on age, aspartate aminotransferase (AST), alanine aminotransferase (ALT) and platelet count, was first proposed to assess liver fibrosis in hepatitis $\mathrm{C}$ virus (HCV)-monoinfected patients [9]. The critical cut-off of FIB-4 for predicting significant liver fibrosis (S2 or greater) is currently set as 1.45 [10]. Recently, it has been reported that the baseline FIB-4 is significantly associated with poor outcomes in patients with liver diseases, such as hepatocellular carcinoma development or all-cause mortality $[11,12]$. FIB- 4 was also reported to be an independent risk factor of chronic kidney disease in patients with non-alcoholic fatty liver disease [13]. The association of FIB-4 with all-cause mortality was also demonstrated in patients with heart failure, which is a non-liver disease [14]. With this report, we searched previous reports on the association between the baseline FIB-4 and all-cause mortality in patients with AAV. However, to our best knowledge, there was no report regarding the association of FIB-4 at the time of diagnosis of AAV with all-cause mortality during follow-up to date. In our retrospective AAV-cohort, allcause mortality was observed only in patients with MPA and GPA, but not EGPA [6]. Hence, in this study, we investigated whether FIB-4 at diagnosis could be associated with all-cause mortality in 132 immunosuppressive drug-naïve patients with MPA and GPA.

\section{Methods}

\section{Patients}

We retrospectively reviewed the medical records of 138 immunosuppressive drug-naïve patients with MPA and GPA based on the following inclusion criteria: i) patients who had been first classified as AAV at the Department of Internal Medicine, Yonsei University College of Medicine, Severance Hospital, from October 2000 to December 2017; ii) patients who fulfilled the American
College of Rheumatology 1990 criteria and were reclassified by the 2007 European Medicines Agency algorithm, in which authors added the modified contents of the Chapel Hill Consensus Conferences (CHCC) Nomenclature of Vasculitis proposed in 2012 [1, 2]; iii) patients who had well-documented medical records with which to assess both clinical manifestations at diagnosis and death during follow-up, and calculate Birmingham vasculitis activity score (BVAS) and FFS (2009) at diagnosis $[15,16]$. Because BVAS for GPA has a different weightsystem compared to BVAS, we evenly applied BVAS to GPA to unify the scoring system; iv) patients who had results on perinuclear (P)-ANCA and cytoplasmic (C)ANCA or myeloperoxidase (MPO)-ANCA and proteinase 3 (PR3)-ANCA levels at diagnosis. v) patients who had been followed up for 12 weeks or greater; vi) patients who had no medical history to affect either BVAS, ANCA positivity or FIB-4 prior to or at diagnosis, particularly, chronic liver diseases including viral hepatitis, coexisting malignancies, serious comorbidities and serious infection, which were identified in the 10th revised International Classification Diseases; vii) patients who had never received immunosuppressive drugs for AAV prior to diagnosis, which were searched by the Korean Drug Utilisation Review system. Of 138 AAV patients, 2 patients were excluded due to $\mathrm{HBsAg}$ positive and one patient was excluded due to anti-HCV positive. And furthermore, 2 patients and one patient were excluded due to alcoholic liver disease and non-alcoholic liver disease, respectively. Finally, we included $132 \mathrm{im}$ munosuppressive drug-naïve patients with MPA $(N=91)$ and GPA $(N=41)$.

\section{Clinical and laboratory data}

We obtained age at diagnosis and gender as demographic data and collected laboratory results including ANCA at diagnosis as described in Table 1. BVAS and FFS (2009) at diagnosis were calculated by reviewing the medical records. Comorbidities belonging to BVAS or FFS (2009), such as interstitial lung disease, diffuse alveolar haemorrhage, gastrointestinal bleeding, cardiovascular diseases, chronic kidney disease $\geq$ stage 3 and cerebrovascular accident, were excluded in AAV-related risk factors for all-cause mortality. In this study, we assessed old age ( $\geq 65$ years), male gender, DM and HTN at diagnosis as conventional risk factors, and GPA, ANCA positivity at diagnosis, BVAS at diagnosis $\geq 16$ and FFS (2009) at diagnosis $\geq 2$ as AAV-related risk factors for all-cause mortality during follow-up according to the previous studies $[6,8]$. We also evaluate the predictive potential of FIB- 4 at diagnosis $\geq 1.45$ for all-cause mortality for the follow-up period. We defined the follow-up duration as the period from diagnosis to the last visit in survived patients, 
Table 1 Baseline characteristics of patients with MPA and GPA $(N=132)$

\begin{tabular}{|c|c|}
\hline Variables & Values \\
\hline \multicolumn{2}{|l|}{ Demographic data } \\
\hline Age at diagnosis (year old) & $57.2 \pm 14.7$ \\
\hline Male gender (N, (\%)) & $40(30.3)$ \\
\hline \multicolumn{2}{|l|}{ AAV variants $(\mathrm{N},(\%))$} \\
\hline MPA & $91(68.9)$ \\
\hline GPA & $41(31.1)$ \\
\hline ANCA positivity $(\mathrm{N},(\%))$ & $112(84.8)$ \\
\hline \multicolumn{2}{|l|}{ Activity and prognostic factor at diagnosis } \\
\hline BVAS & $13.1 \pm 7.2$ \\
\hline FFS (2009) & $1.4 \pm 1.0$ \\
\hline FFS (2009) $\geq 2(N,(\%))$ & $57(43.2)$ \\
\hline \multicolumn{2}{|l|}{ Laboratory results at diagnosis } \\
\hline White blood cell $\left(/ \mathrm{mm}^{3}\right)$ & $9538.3 \pm 3940.5$ \\
\hline Haemoglobin (g/dL) & $12.0 \pm 1.2$ \\
\hline Platelet $\times 10^{3}\left(/ \mathrm{mm}^{3}\right)$ & $331.8 \pm 144.7$ \\
\hline Prothrombin time (INR) & $1.0 \pm 0.1$ \\
\hline Fasting glucose (mg/dL) & $116.8 \pm 44.6$ \\
\hline Blood urea nitrogen (mg/dL) & $28.1 \pm 25.3$ \\
\hline Creatinine $(\mathrm{mg} / \mathrm{dL})$ & $1.9 \pm 2.1$ \\
\hline Protein (g/dL) & $6.7 \pm 0.9$ \\
\hline Serum albumin (g/dL) & $3.5 \pm 0.8$ \\
\hline Alkaline phosphatase (IU/L) & $93.7 \pm 94.3$ \\
\hline Aspartate aminotransferase (IU/L) & $23.2 \pm 26.5$ \\
\hline Alanine aminotransferase (IU/L) & $23.4 \pm 39.3$ \\
\hline Total bilirubin (mg/dL) & $0.7 \pm 1.6$ \\
\hline Total cholesterol (mg/dL) & $171.4 \pm 50.4$ \\
\hline \multicolumn{2}{|l|}{ Acute reactants at diagnosis } \\
\hline Erythrocyte sedimentation rate $(\mathrm{mm} / \mathrm{hr})$ & $62.8 \pm 38.0$ \\
\hline C-reactive protein (mg/L) & $44.0 \pm 56.8$ \\
\hline \multicolumn{2}{|l|}{ Liver fibrosis index at diagnosis } \\
\hline FIB-4 & $1.1 \pm 0.9$ \\
\hline FIB-4 $\geq 1.45(\mathrm{~N},(\%))$ & $27(20.5)$ \\
\hline \multicolumn{2}{|c|}{ Comorbidities except items of BVAS or FFS (2009) at diagnosis ${ }^{\mathrm{a}}(\mathrm{N},(\%))$} \\
\hline $\mathrm{DM}$ & $26(19.7)$ \\
\hline HTN & $61(46.2)$ \\
\hline Follow-up duration (months) & $52.4 \pm 52.2$ \\
\hline Death $(N,(\%))$ & $15(11.4 \%)$ \\
\hline
\end{tabular}

Values are expressed as a mean \pm standard deviation and number $(\mathrm{N})(\%)$ a interstitial lung disease, diffuse alveolar haemorrhage, gastrointestinal bleeding, cardiovascular diseases, chronic kidney disease $\geq$ stage 3 and cerebrovascular accident and so on

AAV ANCA-associated vasculitis, ANCA antineutrophil cytoplasmic antibody, MPA microscopic polyangiitis, GPA granulomatosis with polyangiitis, BVAS Birmingham vasculitis activity score, FFS five factor score, FIB-4 fibrosis-4, DM diabetes mellitus, HTN hypertension whereas we defined it as the period from diagnosis to death in deceased patients.

\section{Equations of FIB-4 and significant liver fibrosis} FIB-4 = age (years) $x$ AST $(\mathrm{IU} / \mathrm{L}) /$ platelet count $\left(10^{9} /\right.$ L)/ $\sqrt{\text { ALT }}$ (IU/L) [9]. The critical cut-off of FIB-4 for significant liver fibrosis (S2-4) was set at 1.45 [10].

\section{Statistical analyses}

All statistical analyses were conducted using SPSS software (version 23 for windows; IBM Corp., Armonk, NY, USA). Continuous variables were expressed as a mean \pm standard deviation, and categorical variables were expressed as a number (percentage). The multivariable Cox hazard model using variables with statistical significance in the univariable Cox hazard model was conducted to appropriately obtain the hazard ratios (HRs) during the considerable follow-up duration. We stratified AAV patients into three groups based on the tertile of BVAS and defined the lower limit of the highest tertile as the cut-off for the current severe AAV (BVAS at diagnosis $\geq 16$ ). The odds ratio (OR) was assessed using the multivariable logistic regression analysis of variables with $p$-values less than 0.05 in the univariable logistic regression analysis. $P$-values less than 0.05 were considered statistically significant.

\section{Results}

Baseline characteristics of 132 patients with MPA and GPA

The baseline characteristics were described in Table 1 . The mean age at diagnosis was 57.2 years and 40 patients $(30.3 \%)$ were men. Ninety-one patients (68.9) were classified as MPA and 41 patients (31.1\%) were as GPA. Any type of ANCA was detected in 112 patients (84.8\%). The mean BVAS and FFS (2009) at diagnosis were 13.1 and 1.4 and 57 patients (43.2\%) had FFS (2009) at diagnosis $\geq 2$. The mean levels of AST and ALT at diagnosis were $23.2 \mathrm{IU} / \mathrm{L}$ and $23.4 \mathrm{IU} / \mathrm{L}$, and the mean platelet count at diagnosis was $331,800.0 / \mathrm{mm}^{3}$. The mean FIB-4 at diagnosis was 1.1 and 27 patients (20.5\%) had significant liver fibrosis (FIB-4 $\geq 1.45$ ). At diagnosis, 26 patients (19.7\%) had DM and 61 patients (46.2\%) had HTN. The mean follow-up duration was 52.4 months and 15 patients (11.4\%) died. During follow-up, glucocorticoid was the most frequently administered drug $(84.8 \%)$, followed by cyclophosphamide $(43.2 \%)$ and azathioprine (24.2\%).

\section{Univariable cox hazards model analysis of risk factors for all-cause mortality}

Among conventional risk factors for all-cause mortality, age at diagnosis $\geq 65$ years (HR 5.055, 95\% confidence interval 1.593, 16.039), DM at diagnosis (HR 3.446, 95\% CI 1.111, 10.692) and HTN at diagnosis (HR 4.611, 95\% 
CI 1.016, 20.927) were significantly associated with allcause mortality Among AAV-related risk factor for allcause mortality, only FFS (2009) at diagnosis $\geq 2$ (HR $4.849,95 \%$ CI $1.341,17.537)$ was significantly associated with all-cause mortality. In addition, FIB-4 at diagnosis $\geq 1.45$ (HR 9.958, 95\% CI 2.550, 38.877) also exhibited a significant association with all-cause mortality during follow-up in patients with MPA and GPA (Table 2).

\section{Multivariable cox hazards model analysis of risk factors for all-cause mortality}

We also conducted the multivariable Cox hazards model using variables with statistical significance in the univariable Cox hazards model. Among age $\geq 65$ years, DM, HTN, FFS (2009) $\geq 2$ and FIB- $4 \geq 1.45$ at diagnosis, only FIB-4 at diagnosis $\geq 1.45$ was an independent predictor of all-cause mortality during the follow-up in patients with MPA and GPA (Table 3).

\section{Discussion}

In this study, we compared the predictive potential of FIB- 4 at diagnosis $\geq 1.45$ with those of conventional and AAV-related risk factors for all-cause mortality in 132 immunosuppressive drug-naïve patients with MPA and GPA. In the multivariable Cox Hazard model analysis, old age ( $\geq 65$ years), DM, HTN, FFS (2009) $\geq 2$ and FIB$4 \geq 1.45$ at diagnosis were included. Interestingly, we found that only FIB- 4 at diagnosis $\geq 1.45$ was associated with all-cause mortality during follow-up in patients MPA and GPA. We considered two reasons why FIB$4 \geq 1.45$ at diagnosis exhibited the relatively high predictive power for all-cause mortality. First, inflammation may accelerate systemic fibrotic change through various inflammatory signals. Thus, the extent of liver fibrosis may indirectly reflect the accumulated amount of

Table 2 Univariable Cox Hazards model analysis of conventional and AAV-related risk factors for all-cause mortality in patients with MPA and GPA

\begin{tabular}{llll}
\hline Variables & HR & $\begin{array}{l}95 \% \text { confidence } \\
\text { interval }\end{array}$ & $P$-value \\
\hline Age at diagnosis $\geq 65$ years & 5.055 & $1.593,16.039$ & 0.006 \\
Male gender & 0.557 & $0.195,1.587$ & 0.273 \\
DM at diagnosis & 3.446 & $1.111,10.692$ & 0.032 \\
HTN at diagnosis & 4.611 & $1.016,20.927$ & 0.048 \\
GPA versus MPA & 1.002 & $0.311,3.226$ & 0.997 \\
ANCA positivity at diagnosis & 3.423 & $0759,15.438$ & 0.109 \\
BVAS at diagnosis $\geq 16$ & 1.554 & $0.553,4.370$ & 0.403 \\
FFS (2009) at diagnosis $\geq 2$ & 4.849 & $1.341,17.537$ & 0.016 \\
FIB-4 at diagnosis $\geq 1.45$ & 9.958 & $2.550,38.877$ & 0.001 \\
\hline
\end{tabular}

$A A V$ ANCA-associated vasculitis, ANCA antineutrophil cytoplasmic antibody, GPA granulomatosis with polyangiitis, MPA microscopic polyangiitis, BVAS Birmingham vasculitis activity score, FFS five factor score, FIB-4 fibrosis-4, DM diabetes mellitus, HTN hypertension
Table 3 Multivariable Cox Hazards model analysis of conventional and AAV-related risk factors for all-cause mortality in patients with MPA and GPA

\begin{tabular}{|c|c|c|c|}
\hline \multirow[t]{2}{*}{ Variables } & \multicolumn{3}{|c|}{ Including age at diagnosis $\geq 65$ years } \\
\hline & $H R$ & $\begin{array}{l}95 \% \text { confidence } \\
\text { interval }\end{array}$ & $P$-value \\
\hline Age at diagnosis $\geq 65$ years & 2.491 & $0.656,9.459$ & 0.180 \\
\hline DM at diagnosis & 1.581 & $0.426,5.864$ & 0.493 \\
\hline HTN at diagnosis & 1.727 & $0.332,8.987$ & 0.516 \\
\hline FFS (2009) at diagnosis $\geq 2$ & 2.921 & $0.705,12.095$ & 0.139 \\
\hline FIB-4 at diagnosis $\geq 1.45$ & 6.253 & $1.398,27.963$ & 0.016 \\
\hline
\end{tabular}

AAV ANCA-associated vasculitis, ANCA antineutrophil cytoplasmic antibody, GPA granulomatosis with polyangiitis, MPA microscopic polyangiitis, BVAS Birmingham vasculitis activity score, FFS five factor score, FIB-4 fibrosis-4, DM diabetes mellitus, HTN hypertension

inflammatory burden in non-liver diseases such as heart failure [14]. We assume that FIB-4 at diagnosis could predict all-cause mortality in MPA and GPA patients in a similar manner. Next, we assume that FIB-4 might be influenced by both conventional and AAV-related risk factors for all-cause mortality prior to or at the time of diagnosis. To prove this assumption, we conducted the univariable and multivariable logistic regression analysis based on FIB-4 at diagnosis $\geq 1.45$ using those risk factors at diagnosis. In the univariable analysis, age at diagnosis $\geq 65$ years (OR 3.812) and DM at diagnosis (OR 5.200) were significantly associated with FIB-4 at diagnosis $\geq 1.45$. BVAS at diagnosis $\geq 16$ and FFS (2009) at diagnosis $\geq 2$ exhibited a tendency to be associated with FIB-4, so they were also included in the multivariable analysis. In the multivariable analysis, only age at diagnosis $\geq 65$ years (OR 3.088, 95\% CI 1.157, 8.239) and $\mathrm{DM}$ at diagnosis (OR 4.556, 95\% CI 1.678, 12.368) were significantly associated with FIB-4 at diagnosis $\geq 1.45$ (Additional file 1: Table S1).

Since, age is one of variables comprising an equation of FIB-4, it is naturally accepted that FIB-4 may be directly correlated with age [9]. Whereas, pre-existing DM could induce non-alcoholic fatty liver disease, one of the systemic complication of DM, which can lead to an increase in FIB-4 within 3 years from baseline [17, 18]. The link between male gender and FIB-4 is controversial: according to a previous review regarding an effect of gender on the outcome of liver diseases, differences in incidence and severity of liver diseases between men and women may vary based on a disease-type [19]. The relation between pre-existing systemic HTN and FIB-4 is also controversial: nonalcoholic fatty liver disease was identified as an independent risk factor for the development of systemic HTN [20]. Therefore, we conclude that FIB-4 at diagnosis itself may be an independent predictor of all-cause mortality in MPA and GPA patients and at 
the same time, it may reflect the effect of old age and $\mathrm{DM}$ on subclinical liver fibrosis, leading to an increase in FIB-4 at diagnosis.

Despite no significant association in the multivariable logistic regression analysis, BVAS and FFS (2009) at diagnosis exhibited a tendency to be associated with FIB-4 at diagnosis. In cases of severe MPA and GPA, as a counterpart of TH1 and TH17 cells, which are important participants in the pathogenesis of AAV, Treg cells may enhance the production of transforming growth factors (TGF)- $\beta$, which can initiate and accelerate liver fibrosis [21]. Moreover, the higher extent of inflammatory burden may provoke the augmented production of reactive oxygen species, which can subsequently promote cell differentiation of fibroblasts to myoblasts in liver [22]. Since BVAS and FFS (2009) were calculated at diagnosis of MPA and GPA, together with FIB-4 at diagnosis, the effect of BVAS and FFS (2009) at diagnosis on FIB-4 at diagnosis might be negligible. However, considering an asymptomatic latency prior to diagnosis of MPA and GPA, BVAS and FFS (2009) at diagnosis might theoretically influence FIB-4 at diagnosis. Therefore, we also conclude that FIB-4 at diagnosis may reflect the subtle effect of the inflammatory burden of AAV on subclinical liver fibrosis, leading to an increase in FIB-4 at diagnosis.

In addition to conventional and AAV-related risk factors, we investigated the predictive potential of comorbidities at diagnosis for all-cause mortality. Interstitial lung disease at diagnosis (HR 6.981, 95\% CI 1.535, 31.756) was significantly associated with all-cause mortality in MPA and GPA patients. However, chronic kidney disease over stage 3 (HR 2.615, 95\% CI 0.883, 7.742), ischaemic heart disease (HR 3.493, 95\% 0.703, 17.361) and cerebrovascular disease (HR 1.213, 95\% CI $0.324,4.546)$ at diagnosis exhibited no significant association with all-cause mortality. We added interstitial lung disease at diagnosis to the multivariable Cox Hazards model analysis together with 5 variables described in Table 3 . Nevertheless, only FIB- 4 at diagnosis $\geq 1.45$ was significantly associated with all-cause mortality. Because interstitial lung disease is not an established conventional or AAV-related risk factor for all-cause mortality, we did not include interstitial lung disease in the multivariable Cox Hazards model in this study.

In this study, we first demonstrated that FIB-4 at diagnosis $\geq 1.45$ is an independent predictor of all-cause mortality during follow-up in patients with MPA and GPA, and furthermore its predictive potential is higher than those of conventional and AAV-related risk factors for all-cause mortality. However, our study also has several issues. First, despite the significant association between FIB-4 at diagnosis and all-cause mortality, we could not clarify the direct mechanism of FIB-4 at diagnosis to estimate all-cause mortality. Second, we could not provide the concrete data on liver fibrosis by liver histology or transient elastography. Third, our study was designed as a retrospective study, we could not strictly control the confounding factors. Particularly, we could not confirm the repeated results of ANCAs in patients without ANCA. Fourth, this study was conducted in a single centre, the number of deceased patients was too small to augment the statistical power. Future prospective and multi-centric studies with a larger number of patients will validate the clinical significance of FIB-4 at diagnosis in predicting all-cause mortality during follow-up of MPA and GPA in real-world clinical practice.

\section{Conclusions}

FIB-4 at diagnosis $\geq 1.45$ is an independent predictor of all-cause mortality during follow-up in patients with MPA and GPA, and furthermore its predictive potential is higher than those of conventional and AAV-related risk factors for all-cause mortality.

\section{Additional file}

Additional file 1: Table S1. Univariable and multivariable logistic regression of conventional and AAV-related risk factors for FIB-4 at diagnosis $\geq 1.45$ in patients with MPA and GPA. (DOCX $20 \mathrm{~kb}$ )

\section{Abbreviations}

AAV: ANCA-associated vasculitis; ALT: Alanine aminotransferase;

ANCA: Antineutrophil cytoplasmic antibody; AST: Aspartate aminotransferase; BVAS: Birmingham vasculitis activity score; C: Cytoplasmic; CHCC: The Chapel Hill Consensus Conferences; DM: Diabetes mellitus; EGPA: Eosinophilic granulomatosis with polyangiitis; FFS: Five factor score; FIB-4: Fibrosis-4; GPA: Granulomatosis with polyangiitis; HCV: Hepatitis C virus;

HTN: Hypertension; MPA: Microscopic polyangiitis; MPO: Myeloperoxidase; P: Perinuclear; PR3: Proteinase 3; TGF: Transforming growth factors

\section{Acknowledgements}

Not applicable.

\section{Authors' contributions}

HJP, JYP, SMJ, JJS, YBP, SWL conceived and designed this study. HJP, JYP, SWL developed methodology. HJP, JYP, SMJ, JJS, YBP, SWL analyzed and interpreted the data. JJS, YBP provided administrative or technical support. HJP, JYP, SWL wrote and/or revised the manuscript. YBP, SWL supervised and coordinated all aspects of the work. All authors have read and approved the manuscript.

\section{Funding}

This research was supported by Basic Science Research Program through the National Research Foundation of Korea (NRF) funded by the Ministry of Education (2017R1D1A1B03029050) to SWL and a grant from the Korea Health Technology R\&D Project through the Korea Health Industry Development Institute, funded by the Ministry of Health and Welfare, Republic of Korea (HI14C1324) to YBP. Funding bodies were not involved in the study design, collection, analysis and interpretation of data in wring the manuscript.

Availability of data and materials

The data used and analysed in this study are available from the corresponding author on reasonable request. 


\section{Ethics approval and consent to participate}

This study was approved by the Institutional Review Board of Severance Hospital (4-2017-0673), who waived the need for patient written informed consent, as this was a retrospective study.

\section{Consent for publication}

Not applicable.

\section{Competing interests}

The authors declare that they have no competing interests.

\section{Author details}

'Division of Rheumatology, Department of Internal Medicine, International St. Mary's Hospital, Catholic Kwandong University College of Medicine, Incheon, Republic of Korea. ${ }^{2}$ Division of Gastroenterology, Department of Internal Medicine, Yonsei University College of Medicine, Seoul, Republic of Korea. ${ }^{3}$ Yonsei Liver Center, Severance Hospital, Seoul, Republic of Korea. ${ }^{4}$ Division of Rheumatology, Department of Internal Medicine, Yonsei University College of Medicine, 50-1 Yonsei-ro, Seodaemun-gu, Seoul 03722, Republic of Korea. ${ }^{5}$ Institute for Immunology and Immunological Diseases, Yonsei University College of Medicine, 50-1 Yonsei-ro, Seodaemun-gu, Seoul 03722, Republic of Korea.

Received: 6 August 2018 Accepted: 31 May 2019

Published online: 13 June 2019

\section{References}

1. Jennette JC, Falk RJ, Bacon PA, Basu N, Cid MC, Ferrario F, et al. 2012 revised international Chapel Hill consensus conference nomenclature of vasculitides. Arthritis Rheum. 2013;65:1-11

2. Watts R, Lane S, Hanslik T, Hauser T, Hellmich B, Koldingsnes W, et al. Development and validation of a consensus methodology for the classification of the ANCA-associated vasculitides and polyarteritis nodosa for epidemiological studies. Ann Rheum Dis. 2007;66:222-7.

3. Millet A, Pederzoli-Ribeil M, Guillevin L, Witko-Sarsat V, Mouthon L. Antineutrophil cytoplasmic antibody-associated vasculitides: is it time to split up the group? Ann Rheum Dis. 2013;72:1273-9.

4. Tan JA, Dehghan N, Chen W, Xie H, Esdaile JM, Avina-Zubieta JA. Mortality in ANCA-associated vasculitis: ameta-analysis of observational studies. Ann Rheum Dis. 2017;76:1566-74

5. Yamagata K, Usui J, Saito C, Yamaguchi N, Hirayama K, Mase K, et al. ANCAassociated systemic vasculitis in Japan: clinical features and prognostic changes. Clin Exp Nephrol. 2012;16:580-8.

6. Mun CH, Yoo J, Jung SM, Song JJ, Park YB, Lee SW. The initial predictors of death in 153 patients with ANCA-associated vasculitis in a single Korean Centre. Clin Exp Rheumatol. 2018:36(Suppl 111):65-72.

7. Córdova-Sánchez BM, Mejía-Vilet JM, Morales-Buenrostro LE, LoyolaRodríguez G, Uribe-Uribe NO, Correa-Rotter R. Clinical presentation and outcome prediction of clinical, serological, and histopathological classification schemes in ANCA-associated vasculitis with renal involvement. Clin Rheumatol. 2016;35:1805-16.

8. Murray CJ, Atkinson C, Bhalla K, Birbeck G, Burstein R, Chou D, et al. The state of US health, 1990-2010: burden of diseases, injuries, and risk factors. JAMA. 2013;310:591-608

9. Vallet-Pichard A, Mallet V, Nalpas B, Verkarre V, Nalpas A, Dhalluin-Venier V, et al. FIB-4: an inexpensive and accurate marker of fibrosis in $\mathrm{HCV}$ infection. Comparison with liver biopsy and fibrotest. Hepatology. 2007;46:32-6.

10. Chou R, Wasson N. Blood tests to diagnose fibrosis or cirrhosis in patients with chronic hepatitis $C$ virus infection: a systematic review. Ann Intern Med. 2013;158:807-20

11. Suh B, Park S, Shin DW, Yun JM, Yang HK, Yu SJ, et al. High liver fibrosis index FIB-4 is highly predictive of hepatocellular carcinoma in chronic hepatitis B carriers. Hepatology. 2015;61:1261-8.

12. Tamaki N, Kurosaki M, Matsuda S, Muraoka M, Yasui Y, Suzuki S, et al. Noninvasive prediction of hepatocellular carcinoma development using serum fibrosis marker in chronic hepatitis C patients. J Gastroenterol. 2014:49:1495-503.

13. Xu HW, Hsu YC, Chang CH, Wei KL, Lin CL. High FIB-4 index as an independent risk factor of prevalent chronic kidney disease in patients with nonalcoholic fatty liver disease. Hepatol Int. 2016;10:340-6.
14. Sato Y, Yoshihisa A, Kanno Y, Watanabe S, Yokokawa T, Abe S, et al. Liver stiffness assessed by Fibrosis-4 index predicts mortality in patients with heart failure. Open Heart. 2017;4:e000598.

15. Mukhtyar C, Lee R, Brown D, Carruthers D, Dasgupta B, Dubey S, et al. Modification and validation of the Birmingham Vasculitis activity score (version 3). Ann Rheum Dis. 2009;68:1827-32.

16. Guillevin L, Pagnoux C, Seror R, Mahr A, Mouthon L, Le Toumelin P, French Vasculitis Study Group (FVSG). The five-factor score revisited: assessment of prognoses of systemic necrotizing vasculitides based on the French Vasculitis study group (FVSG) cohort. Medicine (Baltimore). 2011;90:19-27.

17. Takahashi Y, Kurosaki M, Tamaki N, Yasui Y, Hosokawa T, Tsuchiya K, et al. Non-alcoholic fatty liver disease fibrosis score and FIB-4 scoring system could identify patients at risk of systemic complications. Hepatol Res. 2015; 45:667-75.

18. Giorda CB, Forlani G, Manti R, Mazzotti A, De Cosmo S, Rossi MC, et al. Trend over time in hepatic fibrosis score in a cohort of type 2 diabetes patients. Diabetes Res Clin Pract. 2018;135:65-72.

19. Guy J, Peters MG. Liver disease in women: the influence of gender on epidemiology, natural history, and patient outcomes. Gastroenterol Hepatol (N Y). 2013;9:633-9.

20. Ryoo JH, Suh YJ, Shin HC, Cho YK, Choi JM, Park SK. Clinical association between non-alcoholic fatty liver disease and the development of hypertension. J Gastroenterol Hepatol. 2014;29:1926-31.

21. Wehrens EJ, Prakken BJ, van Wijk F. T cells out of control--impaired immune regulation in the inflamed joint. Nat Rev Rheumatol. 2013;9:34-42.

22. Sampson N, Berger $P$, Zenzmaier C. Redox signaling as a therapeutic target to inhibit myofibroblast activation in degenerative fibrotic disease. Biomed Res Int. 2014;2014:131737.

\section{Publisher's Note}

Springer Nature remains neutral with regard to jurisdictional claims in published maps and institutional affiliations.

Ready to submit your research? Choose BMC and benefit from:

- fast, convenient online submission

- thorough peer review by experienced researchers in your field

- rapid publication on acceptance

- support for research data, including large and complex data types

- gold Open Access which fosters wider collaboration and increased citations

- maximum visibility for your research: over $100 \mathrm{M}$ website views per year

At $\mathrm{BMC}$, research is always in progress.

Learn more biomedcentral.com/submissions 